\title{
Elevated Monocyte to High-density Lipoprotein Ratios as an Inflammation Markers for Schizophrenia Patients
}

\author{
Musa Sahpolat', Duygu Ayar ${ }^{2}$, Mustafa Ari $^{3}$, Mehmet Akif Karaman ${ }^{4}$ \\ ${ }^{1}$ Department of Psychiatry, Kilis State Hospital, ${ }^{2}$ Yusuf Serefoglu Faculty of Health Sciences, Kilis 7 Aralık University, Kilis, ${ }^{3}$ Department of \\ Psychiatry, Mustafa Kemal University Tayfur Ata Sokmen Faculty of Medicine, Hatay, ${ }^{4}$ Department of Psychological Counseling and Guidance, \\ Kilis 7 Aralık University, Kilis, Turkey
}

Objective: Monocyte to high density lipoprotein ratio (MHR) is a new instrument for giving notice inflammation, which plays a main role in schizophrenia. Thus, in this study, our goal was to investigate the possible association between MHR and schizophrenia.

Methods: The participants of this study consisted of 75 schizophrenia patients and 74 healthy individuals (control group). The Positive and Negative Syndrome Scale was used to collect data from the patient group. Complete blood count parameters and lipid profile were analyzed in all study participants.

Results: The patients with schizophrenia had higher MHR values $(15.04 \pm 3.31$ in schizophrenia patients and $12.62 \pm$ 2.99 in controls; $p=0.001$ ). Monocyte counts and MHR of the schizophrenia patients were significantly higher than the control group. There was a significant and positive correlation between age, body mass index, severity of disease and MHR.

Conclusion: To our knowledge, this study was the first to demonstrate inflammatory markers such as MHR levels in schizophrenia patients. Both monocyte counts and MHR values in schizophrenia patients were higher than the control group. MHR might be an available and useful inflammatory marker to evaluate inflammation in schizophrenia patients.

KEY WORDS: High density lipoprotein; Inflammation; Monocyte; Schizophrenia.

\section{INTRODUCTION}

Schizophrenia is a progressive and chronic psychiatric illness influencing roughly $1 \%$ of whole population worldwide $[1,2]$. The average life span of individuals who are diagnosed with schizophrenia is almost 20 years less than healthy individuals without this disorder $[1,2]$. Unfortunately, there is not sufficient information explaining etiology of schizophrenia in the strict sense. One of the important hypotheses to clarify its etiopathogenesis is about immune dysfunction and inflammation [1-3]. Components such as increment in proinflammatory cytokines, increment in autoantibodies, increment oxidative stress

Received: March 29, 2020 / Revised: May 30, 2020

Accepted: June 8, 2020

Address for correspondence: Mustafa Ari

Department of Psychiatry, Mustafa Kemal University Tayfur Ata

Sokmen Faculty of Medicine, Hatay 31115, Turkey

E-mail: drmustafa3142@hotmail.com

ORCID: https://orcid.org/0000-0002-8003-1661 products, and maternal infection in prenatal period strengthen this hypothesis [1-3]. The prevalence of risk factors for cardiovascular disease (CVD), cardiovascular morbidity, and an increasing mortality due to CVD in schizophrenia patients is higher than the general population [2,3]. In addition, metabolic syndrome (MetS) and its related factors are major risk factors for the development of cognitive impairment $[4,5]$. In the study conducted by Zhang and colleagues [6], it was found that schizophrenia patients with MetS had poorer cognitive functions than those without MetS. In addition, same study's results revealed that immune-inflammation might play a major role in this association [6]. Previous studies also indicated the inflammation in MetS had a significant role on cognitive impairment [7]. Hence, identifying biomarkers for inflammation and CVD in schizophrenia patients, and to diminish its occurrence remains as a critical goal.

Monocytes are sources of several cytokines and directly

(c) This is an Open-Access article distributed under the terms of the Creative Commons Attribution Non-Commercial License (http://creativecommons.org/licenses/by-nc/4.0) which permits unrestricted non-commercial use, distribution, and reproduction in any medium, provided the original work is properly cited. 
affect platelets and endothelial cells, which induces proinflammatory and prothrombotic pathways. Monocytes conduct also as an important source of pro-inflammatory species during atherosclerosis process [8-10]. High density lipoprotein (HDL) displays antithrombotic, anti-inflammatory and antioxidant effects [8]. HDL also protects the endothelial tissue from the harmful effects of low-density lipoprotein (LDL), and also prevents the oxidation of LDL $[8,9]$. Decreased HDL levels and increased monocyte counts were also showed to be related with inflammation. So, the monocyte to HDL ratio (MHR) was recommended to be used as a new inflammatory biomarker $[9,10]$. Recent studies showed that MHR might be a new marker of inflammation and oxidative stress. In addition, it was also closely associated to the presence and prognosis of some CVDs [10-12]. As reported in the literature, inflammatory process may be part of etiology of schizophrenia [10-12]. In this study, our goal was to investigate the possible association between MHR and schizophrenia.

\section{METHODS}

This study included a total of 75 schizophrenia patients and 74 randomly selected healthy volunteers admitted to a psychiatric outpatient clinic. The first group consisted of schizophrenia patients diagnosed according to the Diagnostic and Statistical Manual of Mental Disorders 5th edition criteria. The second group consisted of healthy volunteer individuals (control group). The control group was selected by simple random sampling method. Exclusion criteria included: presence of other chronic diseases such as hyperlipidemia, hypertension, DM, asthma, presence of active infection, myocardial infarction, con- gestive heart failure, or neurological disorders, being over the ages of $18-65$, presence of pregnancy.

Socio-demographic datas and body mass index (BMI) were collected. The Positive and Negative Syndrome Scale (PANSS) was used to evaluate the severity of schizophrenia in the patient group [13]. The study protocol was approved by the Institutional Ethics Committee (IRB no. 2020/05). Our study was conducted in accordance with the ethical standards of the responsible committee on human experimentation and the Helsinki Declaration.

Venous blood samples of the individuals were obtained after a fasting period of 12 hours; HDL levels, monocyte counts determined. MHR value was calculated by dividing the monocyte count to HDL level.

\section{Statistical Analysis}

Statistical analysis was performed using the IBM SPSS Statistics 24.0 (IBM Co., Armonk, NY, USA). Descriptive data were expressed as the mean, standard deviation, frequency, and rate. Kolmogorov - Smirnov test was used to test the normality. The independent sample $t$ test for the inter-group comparison of normally distributed quantitative variables was used. Normally distributed qualitative variables were compared using the Pearson's chi-square tests. Pearson's method was used for correlation analysis. $p$ values of $<0.05$ were considered statistically significant.

\section{RESULTS}

The study included 75 (36 females, 39 males) schizophrenia patients with a mean age of $36.91 \pm 10.71$ years and 74 (35 females, 39 males) control individuals with a mean age of $36.65 \pm 6.34$ years $(p>0.05)$ (Table 1$)$.

Table 1. The demographic and biochemical characteristics of two groups

\begin{tabular}{|c|c|c|c|c|}
\hline Variables & Schizophrenia patient group $(n=75)$ & Healthy control group $(n=74)$ & Significance & $p$ value \\
\hline Age (yr) & $36.91 \pm 10.71$ & $36.65 \pm 6.34$ & $0.871^{*}$ & 0.385 \\
\hline Sex (female/male) & $36 / 39$ & $35 / 39$ & $0.007^{* *}$ & 0.932 \\
\hline $\mathrm{BMI}\left(\mathrm{kg} / \mathrm{m}^{2}\right)$ & $24.90 \pm 3.98$ & $24.78 \pm 3.01$ & $0.220^{*}$ & 0.827 \\
\hline Smoking (yes/no) & $36 / 39$ & $31 / 43$ & $0.562^{* *}$ & 0.454 \\
\hline PANSS total score & $87.71 \pm 12.16$ & - & - & - \\
\hline Monocyte $\left(\times 10^{3} / \mu \mathrm{l}\right)$ & $636.57 \pm 98.55$ & $542.97 \pm 96.14$ & $5.867^{*}$ & 0.001 \\
\hline HDL (mg/dl) & $43.49 \pm 7.71$ & $43.97 \pm 6.40$ & $-0.415^{*}$ & 0.697 \\
\hline Monocyte/HDL & $15.04 \pm 3.31$ & $12.62 \pm 2.99$ & $4.660 *$ & 0.001 \\
\hline
\end{tabular}

Values are presented as mean \pm standard deviation or number only. BMI, body mass index; PANSS, Positive and Negative Syndrome Scale; HDL, high density lipoprotein. *Independent Sample $t$ test, ${ }^{* *}$ Pearson's chi-square test, $p<0.05$ is signifiant. 
The mean monocyte counts were $636.57 \pm 98.55 \times 10^{3} / \mu \mathrm{l}$ in schizophrenia patients and $542.97 \pm 96.14 \times 10^{3} / \mu$ in control group, statistically significant difference was found between both groups in terms of mean monocyte counts ( $p=0.001$ and $p<0.05$ ). Although HDL cholesterol levels were lower in schizophrenia patients, but no statistically significant difference was found between both groups ( $p=$ 0.697). MHR of the control group was $12.62 \pm 2.99$ while it was $15.04 \pm 3.31$ in schizophrenia patients. MHR values were found to be statistically significantly higher in schizophrenia patients than control group ( $p=0.001$ and $p<0.05$ ) (Table 1).

In addition, MHR of both groups were significantly and positively correlated with age, BMI and PANSS total scores $(r=0.170, p=0.038 ; r=0.178, p=0.030$ and $r=$ $0.260, p=0.025$, and $p<0.05$, respectively). No statistically significant relationship was found between smoking and MHR in both groups $(p>0.05)$.

\section{DISCUSSION}

Schizophrenic patients are under a high risk of CVD when compared to normal population. Moreover, those patients experience more DM and are at risk of being twice as obese as the normal population. The association between schizophrenia patients and sudden cardiac death has been reported; however, the underlying mechanisms are not sufficiently understood $[1,3]$. The previous studies reported association between schizophrenia and inflammation for a long time [1-3]. The findings related to correlations between schizophrenia and proinflammatory cytokine increase, various infectious diseases, MetS, CVDs and autoimmune diseases have supported the inflammation hypothesis of schizophrenia [1-3].

The main findings of our study were: 1) Elevated MHR was found to be significantly higher in schizophrenia patients, 2) MHR of both groups were significantly and positively correlated with age and BMI, and 3) Significantly positive correlation was found between MHR and severity of disease (PANSS total scores) in schizophrenia patients.

The MHR as a new prognostic factor in CVD has been suggested to be used as an indicator of inflammation. Studies have also indicated that elevated MHR levels were associated with obesity, smoking and muscular bridge diagnoses with coronary anjiography [14-16]. In our study, there was not an evidence of relationship between smok- ing and MHR. However, MHR was significantly and positively correlated with BMI. The activation of monocytes is important and they can be in different forms in the lipid-laden machropages. Both the activated monocytes and these machropages have a significant role promoting the immune system and driving inflammation and CVD $[11,17,18]$. Acikgoz et al. [19] indicated that there was a high and negative relationship between MHR and flowmediated dilatation. Hence, increased MHR might be a useful tool reflecting impaired endothelial function and systemic inflammation. Johnsen et al. [20] reported that an increased monocyte count may be used as an independent predictor of future plaque improvement in already plaque-free arteries. It was stated that the MHR was a significant marker of increased cardiovascular events in chronic renal failure patients, and to be related with a poor prognosis for cardiovascular mortality [21].

Monocytes migrate into tissue macrophages in interaction with platelets and endothelium, which aggravates inflammation [22,23]. The number of monocytes has been shown to predict the premature occurrence of coronary activities, and the activation of monocytes has been a significant process in the onset of atherosclerosis [22-24]. The HDL displays antioxidant, antiinflammatory, and antiplatelet effects by several pathways, such as inhibition of endothelial adhesion protein expression, including contribution to the cholesterol outflow from macrophages, and encouraging reverse transport of oxidized molecules [23-25]. Thus, HDL reduces inflammation via inhibition of monocyte activities and interruption of alteration of monocytes to macrophages $[24,25]$. As a result, combining measurements of HDL and monocyte counts as the MHR might represent the basic inflammatory process.

Studies demonstrated that schizophrenia has increased macrophage/monocyte inflammatory activation pattern. It includes especially monocytosis, high levels of proinflammatory and anti-inflammatory monocyte/macrophage derived cytokines [26-29]. The stimulation of the inflammatory response system can give an idea for activation of microglia cells, as they are the macrophages of the brain $[26,27]$. Another important finding of the study was the prevalence of hypertriglyceridemia and low HDL levels in schizophrenia patients. We found that the prevalence of hypertriglyceridemia (35.2\%) and low HDL levels $(42.6 \%)$ in these patients were higher than in the healthy individuals [28]. Moreover, it was reported that 
acute-phase schizophrenia patients had poorer lipid profiles, such as higher LDL and lower HDL, which are associated with the risk of improving CVD and inflammation [29]. For these reasons, we can say that MHR might be used as an indicator of inflammation in schizophrenia patients.

This study had important findings, which was the first study to investigate MHR values in schizophrenia patients, but there were also some limitations. First, the study included a cross-sectional and retrospective data, and reflected experience of only one psychiatric service. Another limitation was the lack of controlling for antipsychotics used. Therefore, we were unable to compare the effects of antipsychotics drugs on MHR. We believe that the analysis of inflammatuar cytokinesis, such as cortisol, interleukin- 6 as well as MHR would better elucidate the complex relationship between them.

Consequently, to our knowledge, our study was the first to demonstrate inflammatory markers such as MHR levels in schizophrenia patients. Both monocyte counts and MHR values in schizophrenia patients were higher than control individuals in our study population. MHR is a basic, inexpensive instrument that ought to be used for giving notice the systemic inflammatory events in schizophrenia patients.

\section{Acknowledgments}

The authors would like to thank the participants.

\section{Conflicts of Interest}

No potential conflict of interest relevant to this article was reported.

\section{Author Contributions}

Conceptualization: Musa Sahpolat and Mustafa Ari. Data acquisition: Musa Sahpolat. Formal analysis: Musa Sahpolat and Mustafa Ari. Funding: Musa Sahpolat, Duygu Ayar, Mustafa Ari, and Mehmet Akif Karaman. Supervision: Musa Sahpolat, Duygu Ayar, Mustafa Ari, and Mehmet Akif Karaman. Writing - original draft: Musa Sahpolat, Duygu Ayar, Mustafa Ari, and Mehmet Akif Karaman. Writing - review \& editing: Musa Sahpolat, Duygu Ayar, Mustafa Ari, and Mehmet Akif Karaman.

\section{ORCID}

Musa Sahpolat
Duygu Ayar

https://orcid.org/0000-0003-3781-7914

Mustafa Ari

https://orcid.org/0000-0002-8003-1661

Mehmet Akif Karaman

https://orcid.org/0000-0001-7405-5133

\section{REFERENCES}

1. Karadag F, Sengul CB, Enli Y, Karakulah K, Alacam H, Kaptanoglu B, et al. Relationship between serum bilirubin levels and metabolic syndrome in patients with schizophrenia spectrum disorders. Clin Psychopharmacol Neurosci 2017; 15:153-162.

2. Miller BJ, Buckley P, Seabolt W, Mellor A, Kirkpatrick B. Meta-analysis of cytokine alterations in schizophrenia: clinical status and antipsychotic effects. Biol Psychiatry 2011;70: 663-671.

3. Correll CU, Solmi M, Veronese N, Bortolato B, Rosson S, Santonastaso $\mathrm{P}$, et al. Prevalence, incidence and mortality from cardiovascular disease in patients with pooled and specific severe mental illness: a large-scale meta-analysis of $3,211,768$ patients and 113,383,368 controls. World Psychiatry 2017;16:163-180.

4. Qiu C, Fratiglioni L. A major role for cardiovascular burden in age-related cognitive decline. Nat Rev Cardiol 2015;12: 267-277.

5. Kaffashian S, Dugravot A, Elbaz A, Shipley MJ, Sabia S, Kivimäki $M$, et al. Predicting cognitive decline: a dementia risk score vs. the Framingham vascular risk scores. Neurology 2013;80:1300-1306.

6. Zhang C, Fang X, Yao P, Mao Y, Cai J, Zhang Y, et al. Metabolic adverse effects of olanzapine on cognitive dysfunction: a possible relationship between BDNF and TNFalpha. Psychoneuroendocrinology 2017;81:138-143.

7. MacKenzie NE, Kowalchuk C, Agarwal SM, Costa-Dookhan KA, Caravaggio F, Gerretsen P, et al. Antipsychotics, metabolic adverse effects, and cognitive function in schizophrenia. Front Psychiatry 2018;9:622.

8. Barter PJ, Nicholls S, Rye KA, Anantharamaiah GM, Navab M, Fogelman AM. Antiinflammatory properties of HDL. Circ Res 2004;95:764-772.

9. Negi G, Kumar A, Joshi RP, Sharma SS. Oxidative stress and Nrf2 in the pathophysiology of diabetic neuropathy: old perspective with a new angle. Biochem Biophys Res Commun 2011;408:1-5.

10. Abacioglu OO. Monocyte to high-density lipoprotein ratio: a prognostic factor for mitral valve prolapse? Bratisl Lek Listy 2020;121:151-153.

11. Canpolat U, Çetin EH, Cetin S, Aydin S, Akboga MK, Yayla C, et al. Association of monocyte-to-HDL cholesterol ratio with slow coronary flow is linked to systemic inflammation. Clin Appl Thromb Hemost 2016;22:476-482.

12. Kaplan IG, Kaplan M, Abacioglu OO, Yavuz F, Saler T. Monocyte/HDL ratio predicts hypertensive complications. 
Bratis/ Lek Listy 2020;121:133-136.

13. Kay SR, Fiszbein A, Opler LA. The positive and negative syndrome scale (PANSS) for schizophrenia. Schizophr Bull 1987; 13:261-276.

14. Enhos A, Cosansu K, Huyut MA, Turna F, Karacop E, Bakshaliyev N, et al. Assessment of the relationship between monocyte to high-density lipoprotein ratio and myocardial bridge. Arq Bras Cardiol 2019;112:12-17.

15. Vahit D, Akboga MK, Samet Y, Hüseyin E. Assessment of monocyte to high density lipoprotein cholesterol ratio and lymphocyte-to-monocyte ratio in patients with metabolic syndrome. Biomark Med 2017;11:535-540.

16. Yılmaz M, Kayançiçek H. A new inflammatory marker: elevated monocyte to HDL cholesterol ratio associated with smoking. J Clin Med 2018;7:76.

17. Dogan A, Oylumlu M. Increased monocyte-to-HDL cholesterol ratio is related to cardiac syndrome X. Acta Cardiol 2017;72:516-521.

18. Kundi H, Gok M, Kiziltunc E, Cetin M, Cicekcioglu H, Cetin ZG, Karayigit O, Ornek E. Relation between monocyte to high-density lipoprotein cholesterol ratio with presence and severity of isolated coronary artery ectasia. Am J Cardiol 2015;116:1685-1689.

19. Acikgoz N, Kurtoğlu E, Yagmur J, Kapicioglu Y, Cansel M, Ermis N. Elevated monocyte to high-density lipoprotein cholesterol ratio and endothelial dysfunction in Behçet disease. Angiology 2018;69:65-70.

20. Johnsen $\mathrm{SH}$, Fosse E, Joakimsen O, Mathiesen EB, StenslandBugge E, Njølstad I, et al. Monocyte count is a predictor of novel plaque formation: a 7-year follow-up study of 2610 persons without carotid plaque at baseline the Tromsø Study. Stroke 2005;36:715-719.

21. Kanbay M, Solak Y, Unal HU, Kurt YG, Gok M, Cetinkaya H, et al. Monocyte count/HDL cholesterol ratio and cardio- vascular events in patients with chronic kidney disease. Int Urol Nephrol 2014;46:1619-1625.

22. Barter PJ, Baker PW, Rye KA. Effect of high-density lipoproteins on the expression of adhesion molecules in endothelial cells. Curr Opin Lipidol 2002;13:285-288.

23. Açıkgöz SK, Açıkgöz E, Şensoy B, Topal S, Aydoğdu S. Monocyte to high-density lipoprotein cholesterol ratio is predictive of in-hospital and five-year mortality in ST-segment elevation myocardial infarction. Cardiol J 2016;23:505-512.

24. Murphy AJ, Woollard KJ, Hoang A, Mukhamedova N, Stirzaker RA, McCormick SP, et al. High-density lipoprotein reduces the human monocyte inflammatory response. Arterioscler Thromb Vasc Biol 2008;28:2071-2077.

25. Cetin EH, Cetin MS, Canpolat U, Aydin S, Topaloglu S, Aras D, et al. Monocyte/HDL-cholesterol ratio predicts the definite stent thrombosis after primary percutaneous coronary intervention for ST-segment elevation myocardial infarction. Biomark Med 2015;9:967-977.

26. Bergink V, Gibney SM, Drexhage HA. Autoimmunity, in flammation, and psychosis: a search for peripheral markers. Biol Psychiatry 2014;75:324-331

27. Drexhage RC, van der Heul-Nieuwenhuijsen L, Padmos RC, van Beveren N, Cohen D, Versnel MA, et al. Inflammatory gene expression in monocytes of patients with schizophrenia: overlap and difference with bipolar disorder. A study in naturalistically treated patients. Int I Neuropsychopharmacol 2010;13:1369-1381.

28. Huang MC, Lu ML, Tsai CJ, Chen PY, Chiu CC, Jian DL, et al. Prevalence of metabolic syndrome among patients with schizophrenia or schizoaffective disorder in Taiwan. Acta Psychiatr Scand 2009;120:274-280.

29. Huang TL, Chen JF. Serum lipid profiles and schizophrenia: effects of conventional or atypical antipsychotic drugs in Taiwan. Schizophr Res 2005;80:55-59. 